\title{
BreastCore
}

\section{Benign Breast Diseases}

\author{
Marc Thill \\ Department of Gynecology and Gynecological Oncology, Breast Center, AGAPLESION Markus Hospital, Frankfurt/M., Germany
}

The vast majority of lesions in the breast are benign; however, discovering a mass in the breast may cause concerns as well as anxiety for both the patient and the physician since breast cancer is the most common malignancy in women in Western countries. Up to $50 \%$ of all women encounter benign breast problems. Physical examination and breast imaging, including mammography, ultrasound, and magnetic resonance imaging (MRI), assist in the diagnosis of benign breast diseases. Especially the introduction of minimally invasive needle biopsies, such as vacuum-assisted and core biopsy, and their extensive use led to an easy and fast clarification of the lesion without surgery in the majority of patients. Because of the frequency of benign lesions occurring in daily practice, it is important for oncologists, radiologists, and pathologists to distinguish them from premalign lesions and invasive breast cancer, and to assess the patient's risk for developing breast cancer. Therefore, an adequate knowledge and understanding of benign breast diseases is required.

However, in contrast to breast cancer, high-level evidence is not available in order to guide treatment. Management is therefore largely based on the experience/training of the individual physician. In the focus of the current issue of BREAST CARE, the aim is to increase the reader's knowledge in some areas of benign breast diseases. The term 'benign breast diseases' encompasses a large and heterogeneous group of lesions, and some of these may come along with a wide range of symptoms while others are only microscopic incidental findings. The incidence of benign breast diseases starts to increase in the second decade of life and has its peak in the fourth and fifth decade.

Firstly, Paepke et al. [1] focus on benign breast tumors and on their diagnosis and management. They point out how important it is to obtain a proper histologic result by core needle aspiration instead of a cytological result by fine needle aspiration to reach the goal of a discrimination between benign (non-proliferative, prolif- erative without or with atypia) and malignant. Besides the currently used surgical treatment options, the authors also touch on the topic of high-intensity focused ultrasound, which is capable of providing a completely non-invasive treatment without causing damage to the directly adjacent tissues, being guided by ultrasound or MRI.

Secondly, Wolfrum et al. [2] give an excellent overview of the etiology, diagnosis, and treatment of idiopathic granulomatous mastitis. Granulomatous mastitis is a rare benign chronic breast disease, which was first described by Milward in 1970 and then by Kessler and Wolloch in 1972. The exact etiology is still unknown but a variety of hypotheses exist. The most accepted hypothesis is an autoimmune localized response to retained and extravasated fat- and protein-rich secretions of the ducts. The clinical findings can mimic an infectious mastitis or an inflammatory carcinoma. Therefore, precise diagnostics is mandatory. Every one of us is definitely aware of the partially long-lasting and in some cases frustrating treatment that strains both the patient and the therapist. Therefore, the authors provide some distinct recommendations on how to treat this particular illness.

Thirdly, Baumann [3] presents an overview of the conservative and surgical treatment of gynecomastia. Gynecomastia often occurs due to treatment of hypertension and anti-androgenic treatment of prostate cancer. A conservative treatment with the selective estrogen receptor modulator tamoxifen is successful but has side effects. Therefore, the surgical approach is sometimes the patient's preferred option. The surgical techniques are described in the article, and an algorithm for the evaluation and treatment of gynecomastia is also provided.

Obviously, the three articles of this focus only cover a few benign breast diseases, and it is important to continue research and education in this heterogeneous field to learn more about the significance and the value of the different benign breast diseases.

\section{KARGER}

() 2018 S. Karger GmbH, Freiburg

Fax +497614520714
Marc Thill, M.D., Ph.D.

Department of Gynecology and Gynecological Oncology

Breast Center, AGAPLESION Markus Hospita

Wilhelm-Epstein-Straße 4, 60431 Frankfurt, Germany marc.thill@fdk.info 
Some of them, such as atypical hyperplasia (not mentioned in the focus), confer an increase in the patient's future risk of developing breast cancer. Such diseases can be considered as risk markers, rather than premalignant, and should lead to counseling about screening recommendations and risk reduction strategies.

\section{Disclosure Statement}

M.T. has received consultant and speaker honoraria from AstraZeneca, Amgen, Celgene, Genomic Health, Hexal, Medtronic, MSD, Myriad, Neodynamics, Novartis, Pfizer, pfm Medical, Pierre-Fabre, Roche, RTI Surgical, Serag-Wiessner, Surgiceye, Sysmex Europe, Tesaro, and TEVA.

\section{References}

Paepke S, Metz S, Ohlinger R: Benign breast tumours - diagnosis and management. Breast Care 2018;13:DOI: 10.1159/000495919.

2 Wolfrum A, Kümmel A, Theuerkauf I, Pelz I, Reinisch M: Granulomatous mastitis: a therapeutic and diagnostic challenge. Breast Care 2018;13:DOI: 10.1159/000495146.

3 Baumann K: Gynecomastia - conservative and surgical management. Breast Care 2018;13:DOI: 10.1159/000494276. 